\title{
Existence and Asymptotic Behavior of Solutions for Weighted $p(t)$-Laplacian System Multipoint Boundary Value Problems in Half Line
}

\author{
Zhimei Qiu, $^{1}$ Qihu Zhang, ${ }^{1,2}$ and Yan Wang ${ }^{1}$ \\ ${ }^{1}$ School of Mathematical Science, Xuzhou Normal University, Xuzhou, Jiangsu 221116, China \\ ${ }^{2}$ Department of Mathematics and Information Science, Zhengzhou University of Light Industry, \\ Zhengzhou, Henan 450002, China
}

Correspondence should be addressed to Qihu Zhang, zhangqh1999@yahoo.com.cn

Received 5 January 2009; Accepted 20 June 2009

Recommended by Alberto Cabada

This paper investigates the existence and asymptotic behavior of solutions for weighted $p(t)$ Laplacian system multipoint boundary value problems in half line. When the nonlinearity term $f(t, \cdot, \cdot)$ satisfies sub- $\left(p^{-}-1\right)$ growth condition or general growth condition, we give the existence of solutions via Leray-Schauder degree.

Copyright (C) 2009 Zhimei Qiu et al. This is an open access article distributed under the Creative Commons Attribution License, which permits unrestricted use, distribution, and reproduction in any medium, provided the original work is properly cited.

\section{Introduction}

In this paper, we consider the existence and asymptotic behavior of solutions for the following weighted $p(t)$-Laplacian system:

$$
\begin{gathered}
-\Delta_{p(t)} u+\delta f\left(t, u,(w(t))^{1 /(p(t)-1)} u^{\prime}\right)=0, \quad t \in(0,+\infty), \\
u(0)=\lim _{t \rightarrow+\infty} u(t), \quad \lim _{t \rightarrow 0^{+}} w(t)\left|u^{\prime}\right|^{p(t)-2} u^{\prime}(t)=\lim _{t \rightarrow+\infty} w(t)\left|u^{\prime}\right|^{p(t)-2} u^{\prime}(t),
\end{gathered}
$$

where $p \in C([0,+\infty), \mathbb{R}), \quad p(t)>1, \quad \lim _{t \rightarrow+\infty} p(t)$ exists and $\lim _{t \rightarrow+\infty} p(t)>1,-\Delta_{p(t)} u=$ $-\left(w(t)\left|u^{\prime}\right|^{p(t)-2} u^{\prime}\right)^{\prime}$ is called the weighted $p(t)$-Laplacian; $w \in C([0,+\infty), \mathbb{R})$ satisfies $0<w(t)$, for all $t \in(0,+\infty)$, and $(w(t))^{-1 /(p(t)-1)} \in L^{1}(0,+\infty)$; the equivalent $\lim _{r \rightarrow 0^{+}} w(r)\left|u^{\prime}\right|^{p(r)-2} u^{\prime}(r)=\lim _{r \rightarrow+\infty} w(r)\left|u^{\prime}\right|^{p(r)-2} u^{\prime}(r)$ means that $\lim _{r \rightarrow 0^{+}} w(r)\left|u^{\prime}\right|^{p(r)-2} u^{\prime}(r)$ and $\lim _{r \rightarrow+\infty} w(r)\left|u^{\prime}\right|^{p(r)-2} u^{\prime}(r)$ both exist and equal; $\delta$ is a positive parameter. 
The study of differential equations and variational problems with variable exponent growth conditions is a new and interesting topic. Many results have been obtained on these kinds of problems, for example, [1-15]. We refer to [2, 16, 17], the applied background on these problems. If $w(t) \equiv 1$ and $p(t) \equiv p$ (a constant), $-\Delta_{p(t)}$ is the well-known $p$ Laplacian. If $p(t)$ is a general function, $-\Delta_{p(t)}$ represents a nonhomogeneity and possesses more nonlinearity, and thus $-\Delta_{p(t)}$ is more complicated than $-\Delta_{p}$. For example, We have the following.

(1) If $\Omega \subset \mathbb{R}^{n}$ is a bounded domain, the Rayleigh quotient

$$
\lambda_{p(x)}=\inf _{u \in W_{0}^{1, p(x)}(\Omega) \backslash\{0\}} \frac{\int_{\Omega}(1 / p(x))|\nabla u|^{p(x)} d x}{\int_{\Omega}(1 / p(x))|u|^{p(x)} d x}
$$

is zero in general, and only under some special conditions $\lambda_{p(x)}>0$ (see [6]), but the fact that $\lambda_{p}>0$ is very important in the study of $p$-Laplacian problems;

(2) If $w(t) \equiv 1$ and $p(t) \equiv p$ (a constant) and $-\Delta_{p} u>0$, then $u$ is concave; this property is used extensively in the study of one dimensional $p$-Laplacian problems, but it is invalid for $-\Delta_{p(t)}$. It is another difference on $-\Delta_{p}$ and $-\Delta_{p(t)}$.

(3) On the existence of solutions of the following typical $-\Delta_{p(t)}$ problem;

$$
\begin{gathered}
-\left(\left|u^{\prime}\right|^{p(x)-2} u^{\prime}\right)^{\prime}=|u|^{q(x)-2} u+C, \quad x \in \Omega \subset \mathbb{R}^{N}, \\
u=0 \quad \text { on } \partial \Omega,
\end{gathered}
$$

because of the nonhomogeneity of $-\Delta_{p(x)}$, and if $1 \leq \max _{x \in \bar{\Omega}} q(x)<\min _{x \in \bar{\Omega}} p(x)$, then the corresponding functional is coercive, if $\max _{x \in \bar{\Omega}} p(x)<\min _{x \in \bar{\Omega}} q(x)$, then the corresponding functional can satisfy Palais-Smale condition, (see [4, 7]). If $\min _{x \in \bar{\Omega}} p(x) \leq q(x) \leq \max _{x \in \bar{\Omega}} p(x)$, there are more difficulties to testify that the corresponding functional is coercive or satisfying Palais-Smale conditions, and the results on this case are rare.

There are many results on the existence of solutions for $p$-Laplacian equation with multi-point boundary value conditions (see [18-21]). On the existence of solutions for $p(x)$-Laplacian systems boundary value problems, we refer to $[5,7,10-15]$. But results on the existence and asymptotic behavior of solutions for weighted $p(t)$-Laplacian systems with multi-point boundary value conditions are rare. In this paper, when $p(t)$ is a general function, we investigate the existence and asymptotic behavior of solutions for weighted $p(t)$-Laplacian systems with multi-point boundary value conditions. Moreover, the case of $\min _{t \in[0,1]} p(t) \leq q(t) \leq \max _{t \in[0,1]} p(t)$ has been discussed.

Let $N \geq 1$ and $I=[0,+\infty)$; the function $f=\left(f^{1}, \ldots, f^{N}\right): I \times \mathbb{R}^{N} \times \mathbb{R}^{N} \rightarrow \mathbb{R}^{N}$ is assumed to be Caratheodory, by this we mean that

(i) for almost every $t \in I$, the function $f(t, \cdot, \cdot)$ is continuous;

(ii) for each $(x, y) \in \mathbb{R}^{N} \times \mathbb{R}^{N}$, the function $f(\cdot, x, y)$ is measurable on $I$; 
(iii) for each $R>0$, there is a $\beta_{R} \in L^{1}(I, \mathbb{R})$ such that, for almost every $t \in I$ and every $(x, y) \in \mathbb{R}^{N} \times \mathbb{R}^{N}$ with $|x| \leq R,|y| \leq R$, one has

$$
|f(t, x, y)| \leq \beta_{R}(t)
$$

Throughout the paper, we denote

$$
\begin{gathered}
w(0)\left|u^{\prime}\right|^{p(0)-2} u^{\prime}(0)=\lim _{t \rightarrow 0^{+}} w(t)\left|u^{\prime}\right|^{p(t)-2} u^{\prime}(t), \\
w(+\infty)\left|u^{\prime}\right|^{p(+\infty)-2} u^{\prime}(+\infty)=\lim _{t \rightarrow+\infty} w(t)\left|u^{\prime}\right|^{p(t)-2} u^{\prime}(t) .
\end{gathered}
$$

The inner product in $\mathbb{R}^{N}$ will be denoted by $\langle\cdot, \cdot\rangle,|\cdot|$ will denote the absolute value and the Euclidean norm on $\mathbb{R}^{N}$. Let $A C(0,+\infty)$ denote the space of absolutely continuous functions on the interval $(0,+\infty)$. For $N \geq 1$, we set $C=C\left(I, \mathbb{R}^{N}\right), C^{1}=\left\{u \in C \mid u^{\prime} \in\right.$ $C\left((0,+\infty), \mathbb{R}^{N}\right), \lim _{t \rightarrow 0^{+}} w(t)^{1 /(p(t)-1)} u^{\prime}(t)$ exists $\}$. For any $u(t)=\left(u^{1}(t), \ldots, u^{N}(t)\right)$, we denote $\left|u^{i}\right|_{0}=\sup _{t \in(0,+\infty)}\left|u^{i}(t)\right|, \quad\|u\|_{0}=\left(\sum_{i=1}^{N}\left|u^{i}\right|_{0}^{2}\right)^{1 / 2}$ and $\|u\|_{1}=\|u\|_{0}+\left\|(w(t))^{1 /(p(t)-1)} u^{\prime}\right\|_{0}$. Spaces $C$ and $C^{1}$ will be equipped with the norm $\|\cdot\|_{0}$ and $\|\cdot\|_{1}$, respectively. Then $\left(C,\|\cdot\|_{0}\right)$ and $\left(C^{1},\|\cdot\|_{1}\right)$ are Banach spaces. Denote $L^{1}=L^{1}\left(I, \mathbb{R}^{N}\right)$, the norm $\|u\|_{L^{1}}=\left[\sum_{i=1}^{N}\left(\int_{0}^{\infty}\left|u^{i}\right| d t\right)^{2}\right]^{1 / 2}$.

We say a function $u: I \rightarrow \mathbb{R}^{N}$ is a solution of (1.1) if $u \in C^{1}$ with $w(t)\left|u^{\prime}\right|^{p(t)-2} u^{\prime}(t)$ absolutely continuous on $(0,+\infty)$, which satisfies (1.1) almost every on $I$.

In this paper, we always use $C_{i}$ to denote positive constants, if it cannot lead to confusion. Denote

$$
z^{-}=\min _{t \in I} z(t), \quad z^{+}=\max _{t \in I} z(t), \quad \text { for any } z \in C(I, \mathbb{R}) .
$$

We say $f$ satisfies sub- $\left(p^{-}-1\right)$ growth condition, if $f$ satisfies

$$
\lim _{|u|+|v| \rightarrow+\infty}\left(\frac{f(t, u, v)}{(|u|+|v|)^{q(t)-1}}\right)=0, \quad \text { for } t \in I \text { uniformly, }
$$

where $q(t) \in C(I, \mathbb{R})$, and $1<q^{-} \leq q^{+}<p^{-}$. We say $f$ satisfies general growth condition, if we don't know whether $f$ satisfies sub- $\left(p^{-}-1\right)$ growth condition or not.

We will discuss the existence of solutions of (1.1)-(1.2) in the following two cases

(i) $f$ satisfies sub- $\left(p^{-}-1\right)$ growth condition;

(ii) $f$ satisfies general growth condition.

This paper is divided into four sections. In the second section, we will do some preparation. In the third section, we will discuss the existence and asymptotic behavior of solutions of (1.1)-(1.2), when $f$ satisfies sub- $\left(p^{-}-1\right)$ growth condition. Finally, in the fourth section, we will discuss the existence and asymptotic behavior of solutions of (1.1)-(1.2), when $f$ satisfies general growth condition. 


\section{Preliminary}

For any $(t, x) \in I \times \mathbb{R}^{N}$, denote $\varphi(t, x)=|x|^{p(t)-2} x$. Obviously, $\varphi$ has the following properties.

Lemma 2.1 (see [4]). $\varphi$ is a continuous function and satisfies

(i) For any $t \in[0,+\infty), \varphi(t, \cdot)$ is strictly monotone, that is,

$$
\left\langle\varphi\left(t, x_{1}\right)-\varphi\left(t, x_{2}\right), x_{1}-x_{2}\right\rangle>0, \text { for any } x_{1}, x_{2} \in \mathbb{R}^{N}, x_{1} \neq x_{2} \text {. }
$$

(ii) There exists a function $\beta:[0,+\infty) \rightarrow[0,+\infty), \beta(s) \rightarrow+\infty$ as $s \rightarrow+\infty$, such that

$$
\langle\varphi(t, x), x\rangle \geq \beta(|x|)|x|, \quad \forall x \in \mathbb{R}^{N}
$$

It is well known that $\varphi(t, \cdot)$ is a homeomorphism from $\mathbb{R}^{N}$ to $\mathbb{R}^{N}$ for any fixed $t \in$ $[0,+\infty)$. For any $t \in I$, denote by $\varphi^{-1}(t, \cdot)$ the inverse operator of $\varphi(t, \cdot)$, then

$$
\varphi^{-1}(t, x)=|x|^{(2-p(t)) /(p(t)-1)} x, \quad \text { for } x \in \mathbb{R}^{N} \backslash\{0\}, \varphi^{-1}(t, 0)=0 .
$$

It is clear that $\varphi^{-1}(t, \cdot)$ is continuous and sends bounded sets into bounded sets. Let us now consider the following problem with boundary value condition (1.2):

$$
\left(w(t) \varphi\left(t, u^{\prime}(t)\right)\right)^{\prime}=g(t), \quad t \in(0,+\infty),
$$

where $g \in L^{1}$, and satisfies $\int_{0}^{+\infty} g(t) d t=0$. If $u$ is a solution of (2.4) with (1.2), by integrating (2.4) from 0 to $t$, we find that

$$
w(t) \varphi\left(t, u^{\prime}(t)\right)=w(0) \varphi\left(0, u^{\prime}(0)\right)+\int_{0}^{t} g(s) d s
$$

Denote $a=w(0) \varphi\left(0, u^{\prime}(0)\right)$. It is easy to see that $a$ is dependent on $g(t)$. Define operator $F: L^{1} \rightarrow C$ as

$$
F(g)(t)=\int_{0}^{t} g(s) d s, \quad t \in I, g \in L^{1}
$$

By solving for $u^{\prime}$ in (2.5) and integrating, we find that

$$
u(t)=u(0)+F\left\{\varphi^{-1}\left[t,(w(t))^{-1}(a+F(g))\right]\right\}(t), \quad t \in I .
$$

The boundary condition (1.2) implies that

$$
\int_{0}^{+\infty} \varphi^{-1}\left\{t,(w(t))^{-1}[a+F(g)(t)]\right\} d t=0 .
$$


For fixed $h \in C$, we denote

$$
\Lambda_{h}(a)=\int_{0}^{+\infty} \varphi^{-1}\left\{t,(w(t))^{-1}[a+h(t)]\right\} d t .
$$

Throughout the paper, we denote $E=\int_{0}^{+\infty}(w(t))^{-1 /(p(t)-1)} d t$.

Lemma 2.2. The function $\Lambda_{h}(\cdot)$ has the following properties.

(i) For any fixed $h \in C$, the equation

$$
\Lambda_{h}(a)=0
$$

has a unique solution $\tilde{a}(h) \in \mathbb{R}^{N}$.

(ii) The function $\tilde{a}: C \rightarrow \mathbb{R}^{N}$, defined in (i), is continuous and sends bounded sets to bounded sets. Moreover

$$
|\widetilde{a}(h)| \leq 3 N\|h\|_{0}
$$

Proof. (i) From Lemma 2.1, it is immediate that

$$
\left\langle\Lambda_{h}\left(a_{1}\right)-\Lambda_{h}\left(a_{2}\right), a_{1}-a_{2}\right\rangle>0, \quad \text { for } a_{1} \neq a_{2},
$$

and hence, if (2.10) has a solution, then it is unique.

Let $t_{0}=3 N\|h\|_{0}$. If $|a|>t_{0}$, since $(w(t))^{-1 /(p(t)-1)} \in L^{1}(0,+\infty)$ and $h \in C$, it is easy to see that there exists an $i \in\{1, \ldots, N\}$ such that the $i$ th component $a^{i}$ of $a$ satisfies $\left|a^{i}\right|>3\|h\|_{0}$. Thus $\left(a^{i}+h^{i}(t)\right)$ keeps sign on $I$ and

$$
\left|a^{i}+h^{i}(t)\right| \geq\left|a^{i}\right|-\|h\|_{0}>2\|h\|_{0} \quad \text { for any } t \in I,
$$

then

$$
\left|a^{i}+h^{i}(t)\right|^{1 /(p(t)-1)}>\left[2\|h\|_{0}\right]^{1 /(p(\xi)-1)}, \quad \text { where } \xi \in I \text {, for any } t \in I \text {. }
$$

Thus the $i$ th component $\Lambda_{h}^{i}(a)$ of $\Lambda_{h}(a)$ is nonzero and keeps sign, and then we have

$$
\int_{0}^{+\infty} \varphi^{-1}\left\{t,(w(t))^{-1}[a+h(t)]\right\} d t \neq 0 .
$$

Let us consider the equation

$$
\lambda \Lambda_{h}(a)+(1-\lambda) a=0, \quad \lambda \in[0,1]
$$


It is easy to see that all the solutions of (2.16) belong to $b\left(t_{0}+1\right)=\left\{x \in \mathbb{R}^{N}|| x \mid<t_{0}+1\right\}$. So, we have

$$
d_{B}\left[\Lambda_{h}(a), b\left(t_{0}+1\right), 0\right]=d_{B}\left[I, b\left(t_{0}+1\right), 0\right] \neq 0,
$$

and it means the existence of solutions of $\Lambda_{h}(a)=0$.

In this way, we define a function $\tilde{a}(h): C[0,+\infty) \rightarrow \mathbb{R}^{N}$, which satisfies

$$
\Lambda_{h}(\tilde{a}(h))=0
$$

(ii) By the proof of (i), we also obtain $\tilde{a}$ sends bounded sets to bounded sets, and

$$
|\widetilde{a}(h)| \leq 3 N\|h\|_{0}
$$

It only remains to prove the continuity of $\tilde{a}$. Let $\left\{u_{n}\right\}$ be a convergent sequence in $C$ and $u_{n} \rightarrow u$ as $n \rightarrow+\infty$. Since $\left\{\tilde{a}\left(u_{n}\right)\right\}$ is a bounded sequence, then it contains a convergent subsequence $\left\{\tilde{a}\left(u_{n_{j}}\right)\right\}$. Let $\tilde{a}\left(u_{n_{j}}\right) \rightarrow a_{0}$ as $j \rightarrow+\infty$. Since $\Lambda_{u_{n_{j}}}\left(\tilde{a}\left(u_{n_{j}}\right)\right)=0$, letting $j \rightarrow+\infty$, we have $\Lambda_{u}\left(a_{0}\right)=0$. From (i), we get $a_{0}=\tilde{a}(u)$, and it means that $\tilde{a}$ is continuous. This completes the proof.

Now, we define the operator $a: L^{1} \rightarrow \mathbb{R}^{N}$ as

$$
a(u)=\tilde{a}(F(u)) .
$$

It is clear that $a(\cdot)$ is continuous and sends bounded sets of $L^{1}$ to bounded sets of $\mathbb{R}^{N}$, and hence it is a compact continuous mapping.

If $u$ is a solution of (2.4) with (1.2), then

$$
u(t)=u(0)+F\left\{\varphi^{-1}\left[t,(w(t))^{-1}(a(g)+F(g)(t))\right]\right\}(t), \quad \forall t \in[0,+\infty) .
$$

Let us define

$$
\begin{gathered}
P: C^{1} \longrightarrow C^{1}, \quad u \longmapsto u(0) ; \\
Q: L^{1} \longrightarrow \mathbb{R}^{N}, \quad h \longmapsto \int_{0}^{+\infty} h(r) d r ; \\
Q^{*}: L^{1} \longrightarrow L^{1}, \quad h \longmapsto \tau(t) \int_{0}^{+\infty} h(r) d r ;
\end{gathered}
$$


where $\tau \in([0, \infty), R)$ and satisfies $0<\tau(t)<1, t \in I, \int_{0}^{+\infty} \tau(t) d t=1$, and we denote $K_{1}: L^{1} \rightarrow$ $C^{1}$ as

$$
\begin{aligned}
K_{1}(h)(t) & :=\left(K_{1} \circ h\right)(t) \\
& =F\left\{\varphi^{-1}\left[t,(w(t))^{-1}\left(a\left(\left(I-Q^{*}\right) h\right)+F\left(\left(I-Q^{*}\right) h\right)\right)\right]\right\}(t), \quad \forall t \in[0,+\infty) .
\end{aligned}
$$

Lemma 2.3. The operator $K_{1}$ is continuous and sends equi-integrable sets in $L^{1}$ to relatively compact sets in $C^{1}$.

Proof. It is easy to check that $K_{1}(h)(\cdot) \in C^{1}$, for all $h \in L^{1}$. Since $(w(t))^{-1 /(p(t)-1)} \in L^{1}$ and

$$
K_{1}(h)^{\prime}(t)=\varphi^{-1}\left[t,(w(t))^{-1}\left(a\left(\left(I-Q^{*}\right) h\right)+F\left(\left(I-Q^{*}\right) h\right)\right)\right], \quad \forall t \in[0,+\infty),
$$

it is easy to check that $K_{1}$ is a continuous operator from $L^{1}$ to $C^{1}$.

Let now $U$ be an equi-integrable set in $L^{1}$, then there exists $\rho \in L^{1}$, such that

$$
|u(t)| \leq \rho(t) \quad \text { a.e. in } I, \text { for any } u \in L^{1} .
$$

We want to show that $\overline{K_{1}(U)} \subset C^{1}$ is a compact set.

Let $\left\{u_{n}\right\}$ be a sequence in $K_{1}(U)$, then there exists a sequence $\left\{h_{n}\right\} \in U$ such that $u_{n}=K_{1}\left(h_{n}\right)$. For any $t_{1}, t_{2} \in I$, we have that

$$
\left|F\left(\left(I-Q^{*}\right) h_{n}\right)\left(t_{1}\right)-F\left(\left(I-Q^{*}\right) h_{n}\right)\left(t_{2}\right)\right| \leq\left|\int_{t_{1}}^{t_{2}} \rho(t) d t\right|+\left|\int_{t_{1}}^{t_{2}} \tau(t) d t\right| Q \rho .
$$

Hence the sequence $\left\{F\left(\left(I-Q^{*}\right) h_{n}\right)\right\}$ is equicontinuous.

From the definition of $Q^{*}$, we have $F\left(\left(I-Q^{*}\right) h_{n}\right)(+\infty)=0, n=1,2, \ldots$ Thus

$$
\begin{aligned}
\left|F\left(\left(I-Q^{*}\right) h_{n}\right)(t)\right| & =\left|F\left(\left(I-Q^{*}\right) h_{n}\right)(t)-F\left(\left(I-Q^{*}\right) h_{n}\right)(+\infty)\right| \\
& =\left|\int_{t}^{+\infty}\left(\left(I-Q^{*}\right) h_{n}\right)(t) d t\right| \\
& \leq\left|\int_{t}^{+\infty} h_{n}(t) d t\right|+\int_{t}^{+\infty} \tau(t) d t \cdot\left|Q h_{n}\right| \\
& \leq \int_{t}^{+\infty} \rho(t) d t+\int_{t}^{+\infty} \tau(t) d t \cdot Q(\rho) \longrightarrow 0, \text { as } t \longrightarrow+\infty .
\end{aligned}
$$

Thus $\left\{F\left(\left(I-Q^{*}\right) h_{n}\right)\right\}$ is uniformly bounded.

By Ascoli-Arzela theorem, there exists a subsequence of $\left\{F\left(\left(I-Q^{*}\right) h_{n}\right)\right\}$ (which we rename the same) being convergent in $C$. According to the bounded continuous of the operator $a$, we can choose a subsequence of $\left\{a\left(\left(I-Q^{*}\right) h_{n}\right)+F\left(\left(I-Q^{*}\right) h_{n}\right)\right\}$ (which we still denote $\left\{a\left(\left(I-Q^{*}\right) h_{n}\right)+F\left(\left(I-Q^{*}\right) h_{n}\right)\right\}$ is convergent in $C$, then $w(t) \varphi\left(t, K_{1}\left(h_{n}\right)^{\prime}(t)\right)=$ $a\left(\left(I-Q^{*}\right) h_{n}\right)+F\left(\left(I-Q^{*}\right) h_{n}\right)$ is convergent in $C$. 
Since

$$
K_{1}\left(h_{n}\right)(t)=F\left\{\varphi^{-1}\left[r,(w(r))^{-1}\left(a\left(\left(I-Q^{*}\right) h_{n}\right)+F\left(\left(I-Q^{*}\right) h_{n}\right)\right)\right]\right\}(t), \quad \forall t \in[0,+\infty),
$$

from the continuity of $\varphi^{-1}$ and the integrability of $w(t)^{-1 /(p(t)-1)}$ in $L^{1}$, we can see that $K_{1}\left(h_{n}\right)$ is convergent in $C$. Thus that $\left\{u_{n}\right\}$ is convergent in $C^{1}$.

This completes the proof. defined by

We denote by $N_{f}(u):[0,+\infty) \times C^{1} \rightarrow L^{1}$ the Nemytski operator associated to $f$

$$
N_{f}(u)(t)=f\left(t, u(t),(w(t))^{1 /(p(t)-1)} u^{\prime}(t)\right), \quad \text { a.e. on } I
$$

Lemma 2.4. $u$ is a solution of (1.1)-(1.2) if and only if $u$ is a solution of the following abstract equation:

$$
u=P u+Q \delta N_{f}(u)+K_{1}\left(\delta N_{f}(u)\right)
$$

Proof. If $u$ is a solution of (1.1)-(1.2), by integrating (1.1) from 0 to $t$, we find that

$$
w(t) \varphi\left(t, u^{\prime}(t)\right)=a\left(\delta N_{f}(u)\right)+F\left(\delta N_{f}(u)\right)(t), \quad \forall t \in(0,+\infty) .
$$

From (2.31), we have

$u(t)=u(0)+F\left\{\varphi^{-1}\left[t,(w(t))^{-1}\left(a\left(\delta N_{f}\right)+F\left(\delta N_{f}(u)\right)\right)\right]\right\}(t), \quad \forall t \in[0,+\infty)$.

From $w(0)\left|u^{\prime}\right|^{p(0)-2} u^{\prime}(0)=w(+\infty)\left|u^{\prime}\right|^{p(+\infty)-2} u^{\prime}(+\infty)$, we have

$$
\begin{aligned}
Q \delta N_{f}(u) & =0, \\
Q^{*} \delta N_{f}(u) & =0 .
\end{aligned}
$$

So we have

$$
u=P u+Q \delta N_{f}(u)+K_{1}\left(\delta N_{f}(u)\right)
$$

Conversely, if $u$ is a solution of (2.30), then

$$
u(0)=P u+Q \delta N_{f}(u)+K_{1}\left(\delta N_{f}(u)\right)(0)=u(0)+Q \delta N_{f}(u)
$$

Thus $Q \delta N_{f}(u)=0$ and $Q^{*} \delta N_{f}(u)=0$. By the definition of the mapping $a$, we have

$$
K_{1}\left(\delta N_{f}(u)\right)(+\infty)=0
$$


thus

$$
u(+\infty)=P u+Q \delta N_{f}(u)=u(0)
$$

From (2.30), we have

$$
\begin{gathered}
w(t) \varphi\left(t, u^{\prime}\right)=a\left(\left(I-Q^{*}\right) \delta N_{f}\right)+F\left(\left(I-Q^{*}\right) \delta N_{f}(u)\right)(t), \quad \forall t \in(0,+\infty), \\
\left(w(t) \varphi\left(t, u^{\prime}\right)\right)^{\prime}=\left(I-Q^{*}\right) \delta N_{f}(u)(t), \quad \forall t \in(0,+\infty)
\end{gathered}
$$

Obviously $F\left(\left(I-Q^{*}\right) N_{f}(u)\right)(+\infty)=0$, from (2.38), we have

$$
w(0) \varphi\left(0, u^{\prime}(0)\right)=w(+\infty) \varphi\left(+\infty, u^{\prime}(+\infty)\right) .
$$

Since $Q \delta N_{f}(u)=0$, we have $Q^{*} \delta N_{f}(u)=0$ and

$$
\left(w(t) \varphi\left(t, u^{\prime}\right)\right)^{\prime}=\delta N_{f}(u)(t)
$$

Hence $u$ is a solutions of (1.1)-(1.2). This completes the proof.

Lemma 2.5. If $u$ is a solution of (1.1)-(1.2), then for any $j=1, \ldots, N$, there exists an $s^{j} \in(0,+\infty)$ such that $\left(u^{j}\right)^{\prime}\left(s^{j}\right)=0$.

Proof. If it is false, then $u^{j}$ is strictly monotone in $(0,+\infty)$.

(i) If $u^{j}$ is strictly decreasing in $(0,+\infty)$, then $\left(u^{j}\right)(0)>\left(u^{j}\right)(+\infty)$; it is a contradiction to $u(0)=u(+\infty)$.

(ii) If $u^{j}$ is strictly increasing in $(0,+\infty)$, then $\left(u^{j}\right)(0)<\left(u^{j}\right)(+\infty)$; it is a contradiction to $u(0)=u(+\infty)$.

This completes the proof.

\section{3. $f$ Satisfies Sub- $\left(p^{-}-1\right)$ Growth Condition}

In this section, we will apply Leray-Schauder's degree to deal with the existence of solutions for (1.1)-(1.2), when $f$ satisfies sub- $\left(p^{-}-1\right)$ growth condition. Moreover, the asymptotic behavior has been discussed.

Theorem 3.1. Assume that $\Omega$ is an open bounded set in $C^{1}$ such that the following conditions hold.

$\left(1^{0}\right)$ For each $\lambda \in(0,1)$ the problem

$$
\left(w(r)\left|u^{\prime}\right|^{p(r)-2} u^{\prime}\right)^{\prime}=\lambda \delta f\left(r, u,(w(r))^{1 /(p(r)-1)} u^{\prime}\right)
$$

with boundary condition (1.2) has no solution on $\partial \Omega$. 
$\left(2^{0}\right)$ The equation

$$
\omega(a):=\int_{0}^{+\infty} \delta f(t, a, 0) d t=0
$$

has no solution on $\partial \Omega \cap \mathbb{R}^{N}$.

$\left(3^{0}\right)$ The Brouwer degree $d_{B}\left[\omega, \Omega \cap \mathbb{R}^{N}, 0\right] \neq 0$.

Then problems (1.1)-(1.2) have a solution on $\bar{\Omega}$.

Proof. Let us consider the following equation with boundary value condition (1.2):

$$
\left(w(r)\left|u^{\prime}\right|^{p(r)-2} u^{\prime}\right)^{\prime}=\lambda \delta f\left(r, u,(w(r))^{1 /(p(r)-1)} u^{\prime}\right)+(1-\lambda) Q^{*} N_{\delta f}(u), \quad \forall t \in(0,+\infty) .
$$

For any $\lambda \in(0,1]$, observe that if $u$ is a solution to (3.1) with (1.2) or $u$ is a solution to (3.3) with (1.2), we have necessarily

$$
Q N_{\delta f}(u)=0, \quad Q^{*} N_{\delta f}(u)=0 .
$$

It means that (3.1) with (1.2) and (3.3) with (1.2) have the same solutions for $\lambda \in(0,1]$. We denote $N(\cdot, \cdot): C^{1} \times I \rightarrow L^{1}$ defined by

$$
N(u, \lambda)=\lambda N_{\delta f}(u)+(1-\lambda) Q^{*} N_{\delta f}(u),
$$

where $N_{\delta f}(u)$ is defined by (2.29). Let

$$
\begin{aligned}
\Phi_{f}(u, \lambda) & =P u+Q N(u, \lambda)+\left(K_{1} \circ N(u, \lambda)\right) \\
& =P u+Q N_{\delta f}(u)+\left(K_{1} \circ N(u, \lambda)\right)
\end{aligned}
$$

and the fixed point of $\Phi_{f}(u, 1)$ is a solution for (3.3) with (1.2). Also problem (3.3) with (1.2) can be written in the equivalent form

$$
u=\Phi_{f}(u, \lambda)
$$

Since $f$ is Caratheodory, it is easy to see that $N(\cdot, \cdot)$ is continuous and sends bounded sets into equi-integrable sets. It is easy to see that $P$ is compact continuous. According to Lemmas 2.2 and 2.3, we can conclude that $\Phi_{f}(u, \lambda)$ is continuous and compact from $C^{1}$ to $C^{1}$ for any $\lambda \in[0,1]$. We assume that for $\lambda=1$, (3.7) does not have a solution on $\partial \Omega$; otherwise we complete the proof. Now from hypothesis $\left(1^{0}\right)$ it follows that (3.7) has no solutions for $(u, \lambda) \in \partial \Omega \times(0,1]$. For $\lambda=0,(3.3)$ is equivalent to the problem

$$
\left(w(r)\left|u^{\prime}\right|^{p(r)-2} u^{\prime}\right)^{\prime}=Q^{*} N_{\delta f}(u)
$$


and if $u$ is a solution to this problem, we must have

$$
\int_{0}^{+\infty} Q^{*} N_{\delta f}(u) d r=\int_{0}^{+\infty} \delta f\left(r, u(r),(w(r))^{1 /(p(r)-1)} u^{\prime}(r)\right) d r=0
$$

Hence

$$
w(r)\left|u^{\prime}\right|^{p(r)-2} u^{\prime} \equiv c,
$$

where $c \in \mathbb{R}^{N}$ is a constant. From Lemma 2.5, there exist $t_{i} \in(0,+\infty)$, such that $\left(u^{i}\right)^{\prime}\left(t_{i}\right)=0$, $i=1, \ldots, N$. Hence $u^{\prime} \equiv 0$, it holds $u \equiv d$, a constant. Thus by (3.9)

$$
\int_{0}^{+\infty} \delta f(t, d, 0) d r=0
$$

which together with hypothesis $\left(2^{0}\right)$, implies that $u=d \notin \partial \Omega$. Thus we have proved that (3.7) has no solution $(u, \lambda)$ on $\partial \Omega \times[0,1]$, then we get that for each $\lambda \in[0,1]$, the Leray-Schauder degree $d_{\mathrm{LS}}\left[I-\Phi_{f}(\cdot, \lambda), \Omega, 0\right]$ is well defined for $\lambda \in[0,1]$, and from the properties of that degree, we have

$$
d_{\mathrm{LS}}\left[I-\Phi_{f}(\cdot, 1), \Omega, 0\right]=d_{\mathrm{LS}}\left[I-\Phi_{f}(\cdot, 0), \Omega, 0\right]
$$

Now it is clear that the problem

$$
u=\Phi_{f}(u, 1)
$$

is equivalent to problem (1.1)-(1.2), and (3.12) tells us that problem (3.13) will have a solution if we can show that

$$
d_{\mathrm{LS}}\left[I-\Phi_{f}(\cdot, 0), \Omega, 0\right] \neq 0 .
$$

Since

$$
\Phi_{f}(u, 0)=P u+Q N_{\delta f}(u)+K_{1}\left(Q^{*} N_{\delta f}(u)\right),
$$

then

$$
u-\Phi_{f}(u, 0)=u-P u-Q N_{\delta f}(u)-K_{1}(0) .
$$

From Lemma 2.2, we have $K_{1}(0)=F(0)=0$. By the properties of the Leray-Schauder degree, we have

$$
d_{\mathrm{LS}}\left[I-\Phi_{f}(\cdot, 0), \Omega, 0\right]=(-1)^{N} d_{B}\left[\omega, \Omega \cap \mathbb{R}^{N}, 0\right]
$$


where the function $\omega$ is defined in (3.2) and $d_{B}$ denotes the Brouwer degree. By hypothesis $\left(3^{0}\right)$, this last degree is different from zero. This completes the proof.

Our next theorem is a consequence of Theorem 3.1. As an application of Theorem 3.1, let us consider the following equation with (1.2)

$$
\left(w(r)\left|u^{\prime}\right|^{p(r)-2} u^{\prime}\right)^{\prime}=g\left(r, u,(w(r))^{1 /(p(r)-1)} u^{\prime}\right)+e\left(r, u(r),(w(r))^{1 /(p(r)-1)} u^{\prime}(r)\right)
$$

where $e: I \times \mathbb{R}^{N} \times \mathbb{R}^{N} \rightarrow \mathbb{R}^{N}$ is Caratheodory, $g=\left(g^{1}, \ldots, g^{N}\right): I \times \mathbb{R}^{N} \times \mathbb{R}^{N} \rightarrow$ $\mathbb{R}^{N}$ is continuous and Caratheodory, and for any fixed $y_{0} \in \mathbb{R}^{N} \backslash\{0\}$, if $y_{0}^{i} \neq 0$, then $g^{i}\left(r, y_{0}, 0\right) \neq 0$, for all $r \in I$, for all $i=1, \ldots, N$.

Theorem 3.2. Assume that the following conditions hold

$\left(1^{0}\right) g(r, k u, k v)=k^{q(r)-1} g(r, u, v)$ for all $k>0$ and all $(r, u, v) \in I \times \mathbb{R}^{N} \times \mathbb{R}^{N}$, where $q(r) \in C(I, \mathbb{R})$ satisfies $1<q^{-} \leq q^{+}<p^{-} ;$

$\left(2^{0}\right) \lim _{|u|+|v| \rightarrow+\infty}\left(e(r, u, v) /(|u|+|v|)^{q(r)-1}\right)=0$, for $r \in$ I uniformly;

$\left(3^{0}\right)$ for large enough $R_{0}>0$, the equation

$$
\omega_{g}(a):=\int_{0}^{+\infty} g(t, a, 0) d t=0
$$

has no solution on $\partial B\left(R_{0}\right) \cap \mathbb{R}^{N}$, where $B\left(R_{0}\right)=\left\{u \in C^{1} \mid\|u\|_{1}<R_{0}\right\}$;

$\left(4^{0}\right)$ the Brouwer degree $d_{B}\left[\omega_{g}, b\left(R_{0}\right), 0\right] \neq 0$ for large enough $R_{0}>0$, where $b\left(R_{0}\right)=\{x \in$ $\left.\mathbb{R}^{N}|| x \mid<R_{0}\right\}$.

Then problem (3.18) with (1.2) has at least one solution.

Proof. Denote

$$
f\left(r, u,(w(r))^{1 /(p(r)-1)} u^{\prime}, \lambda\right)=g\left(r, u,(w(r))^{1 /(p(r)-1)} u^{\prime}\right)+\lambda e\left(r, u,(w(r))^{1 /(p(r)-1)} u^{\prime}\right) .
$$

At first, we consider the following problem:

$$
\left(w(r)\left|u^{\prime}\right|^{p(r)-2} u^{\prime}\right)^{\prime}=f\left(r, u,(w(r))^{1 /(p(r)-1)} u^{\prime}, \curlywedge\right)
$$

According to the proof of Theorem 3.1, we know that (3.21) with (1.2) has the same solution of

$$
u=\Phi_{f}(u, \lambda)=P u+Q N_{f}(u, \lambda)+K_{1}\left(N_{f}(u, \lambda)\right)
$$

where $N_{f}(u, \lambda)=f\left(r, u,(w(r))^{1 /(p(r)-1)} u^{\prime}, \lambda\right)$.

We claim that all the solutions of (3.21) are uniformly bounded for $\lambda \in[0,1]$. In fact, if it is false, we can find a sequence of solutions $\left\{\left(u_{n}, \lambda_{n}\right)\right\}$ for (3.21) with (1.2) such that $\left\|u_{n}\right\|_{1} \rightarrow+\infty$ as $n \rightarrow+\infty$, and $\left\|u_{n}\right\|_{1}>1$ for any $n=1,2, \ldots$ 
Journal of Inequalities and Applications

Since $\left(u_{n}, \lambda_{n}\right)$ are solutions of (3.21) with (1.2), so $u_{n}(0)=u_{n}(+\infty)$. According to Lemma 2.5, there exist $\xi_{n}^{i_{n}} \in(0,+\infty)$ such that $\left(u_{n}^{i_{n}}\right)^{\prime}\left(\xi_{n}^{i_{n}}\right)=0$, then

$$
\begin{gathered}
w(t)\left|u_{n}^{\prime}\right|^{p(t)-2}\left(u_{n}^{i_{n}}\right)^{\prime}(t)=\int_{\xi_{n}^{i_{n}}}^{t} f^{i_{n}}\left[r, u_{n},(w(r))^{1 /(p(r)-1)} u_{n}^{\prime}, \lambda_{n}\right] d r, \quad \forall t \in(0,+\infty), \\
\begin{aligned}
w(t)\left|u_{n}^{\prime}\right|^{p(t)-2}\left(u_{n}^{i_{n}}\right)^{\prime}(t)=\int_{\xi_{n}^{i_{n}}}^{t}\left\|u_{n}\right\|_{1}^{q(r)-1}\left\{g^{i_{n}}\left[r, \frac{u_{n}}{\left\|u_{n}\right\|_{1}}, \frac{(w(r))^{1 /(p(r)-1)} u_{n}^{\prime}}{\left\|u_{n}\right\|_{1}}\right]\right. \\
+o(1)\} d r, \quad \forall t \in(0,+\infty),
\end{aligned}
\end{gathered}
$$

where $o(1)$ means the function which is uniformly convergent to 0 (as $n \rightarrow+\infty$ ). According to the property of $g$ and (3.23), then there exists a positive constant $C_{1}$ such that

$$
w(t)\left|u_{n}^{\prime}\right|^{p(t)-2}\left|\left(u_{n}^{i_{n}}\right)^{\prime}(t)\right| \leq C_{1}\left\|u_{n}\right\|_{1}^{q^{+}-1}, \quad \forall t \in(0,+\infty),
$$

then we have

$$
w(t)\left|u_{n}^{\prime}(t)\right|^{p(t)-1} \leq 2 N C_{1}\left\|u_{n}\right\|_{1}^{q^{+}-1}, \quad \forall t \in(0,+\infty) .
$$

Denote $\alpha=\left(q^{+}-1\right) /\left(p^{-}-1\right)$, then

$$
\sup _{t \in(0,+\infty)}\left|(w(t))^{1 /(p(t)-1)} u_{n}^{\prime}(t)\right| \leq C_{2}\left\|u_{n}\right\|_{1}^{\alpha} .
$$

Thus

$$
\left\|(w(t))^{1 /(p(t)-1)} u_{n}^{\prime}(t)\right\|_{0} \leq N C_{2}\left\|u_{n}\right\|_{1}^{\alpha} .
$$

Since $\alpha \in(0,1)$, from (3.27) we have

$$
\lim _{n \rightarrow+\infty} \frac{\left\|u_{n}\right\|_{0}}{\left\|u_{n}\right\|_{1}}=1
$$

Denote $b_{n}=\left(\left|u_{n}^{1}\right|_{0} /\left\|u_{n}\right\|_{0},\left|u_{n}^{2}\right|_{0} /\left\|u_{n}\right\|_{0}, \ldots,\left|u_{n}^{N}\right|_{0} /\left\|u_{n}\right\|_{0}\right)$, then $b_{n} \in \mathbb{R}^{N}$ and $\left|b_{n}\right|=1(n=$ $1,2, \ldots$ ), then $\left\{b_{n}\right\}$ possesses a convergent subsequence (which denoted by $b_{n}$ ), and then there exists a vector $b_{0}=\left(b_{0}^{1}, b_{0}^{2}, \ldots, b_{0}^{N}\right) \in \mathbb{R}^{N}$ such that

$$
\left|b_{0}\right|=1, \quad \lim _{n \rightarrow+\infty} b_{n}=b_{0} .
$$


Without loss of generality, we assume that $b_{0}^{1}>0$. Since $u_{n} \in C(I, \mathbb{R})$, there exist $\eta_{n}^{i} \in$ $(0,+\infty)$ such that

$$
\left|u_{n}^{i}\left(\eta_{n}^{i}\right)\right| \geq\left(1-\frac{1}{2 n}\right)\left|u_{n}^{i}\right|_{0^{\prime}} \quad i=1,2, \ldots, N ; n=1,2, \ldots
$$

and then from (3.27) we have

$$
0 \leq\left|u_{n}^{1}(r)-u_{n}^{1}\left(\eta_{n}^{1}\right)\right|=\left|\int_{\eta_{n}^{1}}^{r}\left(u_{n}^{1}\right)^{\prime}(t) d t\right| \leq C_{2}\left\|u_{n}\right\|_{1}^{\alpha} \int_{0}^{+\infty}(w(t))^{-1 /(p(t)-1)} d t .
$$

Since $\left\|u_{n}\right\|_{1} \rightarrow+\infty($ as $n \rightarrow+\infty), \alpha \in(0,1)$, and $b_{0}^{1}>0$, we have

$$
\lim _{n \rightarrow+\infty} \frac{1}{\left|u_{n}^{1}\left(\eta_{n}^{1}\right)\right|} C_{2}\left\|u_{n}\right\|_{1}^{\alpha} \int_{0}^{+\infty}(w(t))^{-1 /(p(t)-1)} d t=0
$$

From (3.28)-(3.32), we have

$$
\lim _{n \rightarrow+\infty} \frac{u_{n}^{1}(r)}{u_{n}^{1}\left(\eta_{n}^{1}\right)}=1, \quad \text { for } r \in I \text { uniformly. }
$$

So we get

$$
\lim _{n \rightarrow+\infty} \frac{u_{n}(r)}{\left\|u_{n}\right\|_{1}}=b_{* \prime}, \quad \lim _{n \rightarrow+\infty} \frac{(w(r))^{1 /(p(r)-1)} u_{n}^{\prime}(r)}{\left\|u_{n}\right\|_{1}}=0, \quad \text { for } r \in I \text { uniformly, }
$$

where $b_{*} \in \mathbb{R}^{N}$, satisfies $\left|b_{*}\right|=1,\left|b_{*}^{i}\right|=b_{0}^{i}$.

Since $b_{0}^{1} \neq 0$, from(1.2) and (3.34), we have

$$
0=\int_{0}^{+\infty}\left\|u_{n}\right\|_{1}^{q(t)-1}\left\{g^{1}\left[t, b_{*}+o(1), o(1)\right]+o(1)\right\} d t
$$

Since $g^{1}\left(t, b_{*}, 0\right) \neq 0$, according to the continuity of $g^{1}$, we have

$$
\int_{0}^{+\infty}\left\|u_{n}\right\|_{1}^{q(t)-1}\left\{g^{1}\left[t, b_{*}+o(1), o(1)\right]+o(1)\right\} d t \neq 0
$$

and it is a contradiction to (3.35). This implies that there exists a big enough $R_{0}>0$ such that all the solutions of (3.21) with (1.2) belong to $B\left(R_{0}\right)$, and then we have

$$
d_{\mathrm{LS}}\left[I-\Phi_{f}(\cdot, 1), B\left(R_{0}\right), 0\right]=d_{\mathrm{LS}}\left[I-\Phi_{f}(\cdot, 0), B\left(R_{0}\right), 0\right] .
$$

If we prove that $d_{\mathrm{LS}}\left[I-\Phi_{f}(\cdot, 0), B\left(R_{0}\right), 0\right] \neq 0$, then we obtain the existence of solutions (3.18) with (1.2). 
Now we consider the following equation with: (1.2)

$$
\left(w(r)\left|u^{\prime}\right|^{p(r)-2} u^{\prime}\right)^{\prime}=\lambda g\left(r, u,(w(r))^{1 /(p(r)-1)} u^{\prime}\right)+(1-\lambda) Q^{*} N_{g}(u),
$$

where $N_{g}(u)=g\left(r, u,(w(r))^{1 /(p(r)-1)} u^{\prime}\right)$.

We denote $\widetilde{N}(\cdot, \cdot): C^{1} \times I \rightarrow L^{1}$ defined by

$$
\widetilde{N}(u, \lambda)=\lambda N_{g}(u)+(1-\lambda) Q^{*} N_{g}(u)
$$

Similar to the proof of Theorem 3.1, we know that (3.38) with (1.2) has the same solution of

$$
u=\Phi_{g}(u, \lambda)=P u+Q \widetilde{N}(u, \lambda)+K_{1}(\widetilde{N}(u, \lambda)) .
$$

Similar to the discussions of the above, for any $\lambda \in(0,1]$, all the solutions of (3.38) with (1.2) are uniformly bounded.

If $u$ is a solution of the following equation with (1.2):

$$
\left(w(r)\left|u^{\prime}\right|^{p(r)-2} u^{\prime}\right)^{\prime}=Q^{*} N_{g}(u),
$$

then we have

$$
\begin{gathered}
Q^{*} N_{g}(u)=0, \\
w(r)\left|u^{\prime}\right|^{p(r)-2} u^{\prime} \equiv c .
\end{gathered}
$$

Since $u(0)=u(+\infty)$, we have $w(r)\left|u^{\prime}\right|^{p(r)-2} u^{\prime} \equiv 0$, and it means that $u$ is a solution of

$$
\omega_{g}(a)=\int_{0}^{+\infty} g(t, a, 0) d t=0
$$

according to hypothesis $\left(3^{0}\right),(3.38)$ has no solutions $(u, \lambda)$ on $\partial B\left(R_{0}\right) \times[0,1]$, then we get that for each $\lambda \in[0,1]$, the Leray-Schauder degree $d_{\mathrm{LS}}\left[I-\Phi_{g}(\cdot, \lambda), B\left(R_{0}\right), 0\right]$ is well defined, and from the properties of that degree, we have

$$
d_{\mathrm{LS}}\left[I-\Phi_{g}(\cdot, 1), B\left(R_{0}\right), 0\right]=d_{\mathrm{LS}}\left[I-\Phi_{g}(\cdot, 0), B\left(R_{0}\right), 0\right]
$$

Now it is clear that $\Phi_{g}(u, 1)=\Phi_{f}(u, 0)$. So $d_{\mathrm{LS}}\left[I-\Phi_{g}(\cdot, 1), B\left(R_{0}\right), 0\right]=d_{\mathrm{LS}}[I-$ $\left.\Phi_{f}(\cdot, 0), B\left(R_{0}\right), 0\right]$. If we prove that $d_{\mathrm{LS}}\left[I-\Phi_{g}(\cdot, 0), B\left(R_{0}\right), 0\right] \neq 0$, then we obtain the existence of solutions (3.18) with (1.2). By the properties of the Leray-Schauder degree, we have

$$
d_{\mathrm{LS}}\left[I-\Phi_{g}(\cdot, 0), B\left(R_{0}\right), 0\right]=(-1)^{N} d_{B}\left[\omega_{g}, b\left(R_{0}\right), 0\right] .
$$


By hypothesis $\left(4^{0}\right)$, this last degree is different from zero. We obtain that (3.18) with (1.2) has at least one solution. This completes the proof.

Corollary 3.3. If $e: I \times \mathbb{R}^{N} \times \mathbb{R}^{N} \rightarrow \mathbb{R}^{N}$ is Caratheodory, which satisfies the conditions of Theorem 3.2, $g(r, u, v)=\beta(r)\left(|u|^{q(r)-2} u+|v|^{q(r)-2} v\right)$, where $\beta(r) \in L^{1}(I, \mathbb{R}), \beta(r), q(r) \in C(I, \mathbb{R})$ are positive functions, and satisfies $1<q^{-} \leq q^{+}<p^{-}$; then (3.18) with (1.2) has at least one solution.

Proof. Since

$$
\begin{gathered}
g(r, u, v)=\beta(r)\left(|u|^{q(r)-2} u+|v|^{q(r)-2} v\right), \\
\omega_{g}(a)=\int_{0}^{+\infty} g(t, a, 0) d t=\int_{0}^{+\infty} \beta(t)|a|^{q(t)-2} a d t
\end{gathered}
$$

then $\omega_{g}(a)=0$ has only one solution $a=0$, and

$$
d_{B}\left[\omega_{g}, b\left(R_{0}\right), 0\right]=d_{B}\left[I, b\left(R_{0}\right), 0\right] \neq 0,
$$

and according to Theorem 3.2, we get that (3.18) with (1.2) has at least a solution. This completes the proof.

Now let us consider the boundary asymptotic behavior of solutions of system (1.1)-

Theorem 3.4. If $u$ is a solution of (1.1)-(1.2) which is given in Theorem 3.2, then

(i) $\left|u^{\prime}(t)\right| \leq C_{1} / w(t)^{1 /(p(t)-1)}, t \in(0,+\infty)$;

(ii) $|u(+\infty)-u(r)| \leq \int_{r}^{+\infty}\left(C_{2} / w(t)^{1 /(p(t)-1)}\right) d t$, as $r \rightarrow+\infty$;

(iii) $|u(r)-u(0)| \leq \int_{0}^{r}\left(C_{3} / w(t)^{1 /(p(t)-1)}\right) d t$, as $r \rightarrow 0^{+}$.

Proof. Since $\lim _{r \rightarrow+\infty} p(r)$ exists and $\lim _{r \rightarrow+\infty} p(r)>1, \lim _{t \rightarrow 0^{+}} w(t)\left|u^{\prime}\right|^{p(t)-2} u^{\prime}(t)$ and $\lim _{t \rightarrow+\infty} w(t)$ $\times\left|u^{\prime}\right|^{p(t)-2} u^{\prime}(t)$ both exist and equal, we can conclude that $\lim _{t \rightarrow 0^{+}} w(t)^{1 /(p(t)-1)} u^{\prime}(t)=$ $\lim _{t \rightarrow+\infty} w(t)^{1 /(p(t)-1)} u^{\prime}(t)$. Since $u \in C^{1}$, we have $\left|w(t)^{1 /(p(t)-1)} u^{\prime}(t)\right| \leq C$, for all $t \in[0,+\infty)$. Thus

(i) $\left|u^{\prime}(t)\right| \leq C_{1} / w(t)^{1 /(p(t)-1)}, t \in(0,+\infty)$;

(ii) $|u(+\infty)-u(r)|=\left|\int_{r}^{+\infty} u^{\prime}(t) d t\right| \leq \int_{r}^{+\infty}\left(C_{2} / w(t)^{1 /(p(t)-1)}\right) d t$, as $r \rightarrow+\infty$;

(iii) $|u(r)-u(0)|=\left|\int_{0}^{r} u^{\prime}(t) d t\right| \leq \int_{0}^{r}\left(C_{3} / w(t)^{1 /(p(t)-1)}\right) d t$, as $r \rightarrow 0^{+}$.

This completes the proof.

Corollary 3.5. Assume that $\lim _{r \rightarrow+\infty} p(r)$ exists, $\lim _{r \rightarrow+\infty} p(r)>1$, and

$$
\begin{aligned}
& C_{4} \leq \frac{w(t)}{t^{\alpha}} \leq C_{5}, \quad \alpha>p(t)-1 \quad \text { as } t \longrightarrow+\infty, \\
& C_{6} \leq \frac{w(t)}{t^{\alpha}} \leq C_{7}, \quad \alpha<p(t)-1 \quad \text { as } t \longrightarrow 0^{+},
\end{aligned}
$$


then

(i) $\left|u^{\prime}(t)\right| \leq C_{8} / t^{\alpha /(p(t)-1)}, t \in(0,+\infty)$;

(ii) $|u(+\infty)-u(r)| \leq \int_{r}^{+\infty}\left(C_{9} / t^{\alpha /(p(t)-1)}\right) d t$, as $r \rightarrow+\infty$;

(iii) $|u(r)-u(0)| \leq \int_{0}^{r}\left(C_{10} / t^{\alpha /(p(t)-1)}\right) d t$, as $r \rightarrow 0^{+}$.

\section{4. $f$ Satisfies General Growth Condition}

In this section, under the condition that $f=\left(f^{1}, \ldots, f^{N}\right)$ satisfies

$$
f^{i}(r, x, y)=\Theta^{i}(r)\left\{\sigma(r)\left(|x|^{q_{1}(r)-1} x^{i}+\mu^{i}(r)|y|^{q_{2}(r)}\right)+e^{i}(r)\right\}
$$

where $q_{1}, q_{2} \in L^{\infty}(I, \mathbb{R})$ are nonnegative, $\sigma \in C^{1}(I, \mathbb{R}), \mu, e \in L^{\infty}\left(I, \mathbb{R}^{N}\right), \Theta^{i} \in L^{1}$ and $\Theta^{i}(r)>0$, almost every in $(0,+\infty), r \in I$, we will apply Leray-Schauder's degree to deal with the existence of solutions for (1.1) with boundary value problems. Moreover the asymptotic behavior has been discussed.

Throughout the paper, assume that

$\left(\mathrm{A}_{1}\right) q_{1}, q_{2} \in L^{\infty}(I, \mathbb{R})$ are nonnegative and satisfying essinf $\left(q_{1}(r)-q_{2}(r)\right)>0$ or $\operatorname{essinf}\left(q_{2}(r)-q_{1}(r)\right)>0 ;$

$\left(\mathrm{A}_{2}\right) \mu=\left(\mu^{1}, \ldots, \mu^{N}\right) \in L^{\infty}\left(I, \mathbb{R}^{N}\right) ; e=\left(e^{1}, \ldots, e^{N}\right) \in L^{\infty}\left(I, \mathbb{R}^{N}\right) ; \sigma$ keeps sign on $I$, and satisfies

$$
\sigma_{1} \leq \underset{r \in I}{\operatorname{essinf}}|\sigma(r)| \leq \underset{r \in I}{\operatorname{esssup}}|\sigma(r)| \leq \sigma_{2}
$$

where $\sigma_{1}$ and $\sigma_{2}$ are positive constants.

For any $h \in L^{\infty}\left(I, \mathbb{R}^{N}\right)$, without loss of generality, we may denote $\left|h^{i}\right|_{0}=$ $\operatorname{esssup}_{r \in(0,+\infty)}|h(r)|(i=1, \ldots, N),\|h\|_{0}=\left(\sum_{i=1}^{N}\left|h^{i}\right|_{0}^{2}\right)^{1 / 2}$. Denote $\theta=\varepsilon /(2+1 / E)$. According to $\left(\mathrm{A}_{1}\right)$, then there exists a positive constant $\varepsilon$ that satisfies

$$
b_{1}:=\underset{r \in I}{\operatorname{essinf}}\left(\frac{E}{N(2 E+1)}|\theta|^{q_{1}(t)}-\|\mu\|_{0}|N \varepsilon|^{q_{2}(t)}\right)>0
$$

We also assume the following

$\left(\mathrm{A}_{3}\right) e=\left(e^{1}, \ldots, e^{N}\right)$ satisfies

$$
\left|e^{i}\right|_{0}<\sigma_{1} b_{1}, \quad i=1, \ldots, N
$$

$\left(\mathrm{A}_{4}\right) \sigma_{2}$ satisfies

$$
\sigma_{2}<b_{2}:=\frac{\inf _{t \in I}|\varepsilon / 2(E+1)|^{p(t)-1}}{2 N(E+1) \int_{0}^{+\infty} \sum_{i=1}^{N} \Theta^{i}(t)\left(|N \varepsilon|^{q_{1}(t)}+\|\mu\|_{0}|N \varepsilon|^{q_{2}(t)}+|\theta|^{q_{1}(t)}\right) d t} .
$$


Note 1. Let $f^{i}(r, x, y)=\lambda \Theta^{i}(r)\left\{\sigma(r)\left[\left(|x|^{q_{1}(r)-1} x^{i}+\mu^{i}(r)|y|^{q_{2}(r)}\right)+\delta e^{i}(r)\right]\right\}$, and $\left(\mathrm{A}_{1}\right)-\left(\mathrm{A}_{2}\right)$ are satisfied. If $\lambda$ and $\delta$ are positive small enough, then it is easy to see that $\left(\mathrm{A}_{3}\right)-\left(\mathrm{A}_{4}\right)$ are satisfied.

Denote

$$
\Omega_{\varepsilon}=\left\{u \in C^{1} \mid \max _{1 \leq i \leq N}\left(\left|u^{i}\right|_{0}+\left|(w(r))^{1 /(p(r)-1)}\left(u^{i}\right)^{\prime}\right|_{0}\right)<\varepsilon\right\} .
$$

It is easy to see that $\Omega_{\varepsilon}$ is an open bounded domain in $C^{1}$.

Theorem 4.1. If $f$ satisfies (4.1), and $\left(A_{1}\right)-\left(A_{4}\right)$ are satisfied, then the system (1.1)-(1.2) has a solution on $\overline{\Omega_{\varepsilon}}$.

Proof. We only need to prove that the conditions of Theorem 3.1 are satisfied.

$\left(1^{0}\right)$ We only need to prove that for each $\lambda \in(0,1)$ the problem

$$
\left(w(r)\left|u^{\prime}\right|^{p(r)-2} u^{\prime}\right)^{\prime}=\lambda f\left(r, u,(w(r))^{1 /(p(r)-1)} u^{\prime}\right)
$$

with boundary condition (1.2) has no solution on $\partial \Omega_{\varepsilon}$.

If it is false, then there exists a $\lambda \in(0,1)$ and $u \in \partial \Omega_{\varepsilon}$ is a solution of (4.7) with (1.2).

Since $u \in \partial \Omega_{\varepsilon}$, there exists an $i$ such that $\left|u^{i}\right|_{0}+\left|(w(r))^{1 /(p(r)-1)}\left(u^{i}\right)^{\prime}\right|_{0}=\varepsilon$.

(i) Suppose that $\left|u^{i}\right|_{0}>2 \theta=2 \varepsilon /(2+1 / E)$, then $\left|(w(r))^{1 /(p(r)-1)}\left(u^{i}\right)^{\prime}\right|_{0}<\varepsilon-2 \theta=\theta / E$. Since $u \in C$, there exists $r_{0} \in I$ such that $\left|u^{i}\left(r_{0}\right)\right|>2 \theta$. For any $r \in I$, we have

$$
\begin{aligned}
\left|u^{i}(r)-u^{i}\left(r_{0}\right)\right| & =\left|\int_{r_{0}}^{r}\left(u^{i}(t)\right)^{\prime} d t\right| \leq \int_{0}^{+\infty}(w(t))^{-1 /(p(t)-1)}\left|(w(t))^{1 /(p(t)-1)}\left(u^{i}(t)\right)^{\prime}\right|_{0} d t \\
& \leq E \cdot \frac{\theta}{E}=\theta .
\end{aligned}
$$

This implies that $\left|u^{i}(r)\right|>\theta$ for each $r \in I$. Since $u \in C, u^{i}(r)$ keeps sign. Since $\sigma(r)$ keeps sign, $\sigma(r) u^{i}(r)$ also keeps sign.

Assume that $\sigma u^{i}$ is positive, then

$$
\begin{aligned}
& f^{i}\left(r, u,(w(r))^{1 /(p(r)-1)} u^{\prime}\right) \\
& \quad \geq \Theta^{i}(r)\left\{|\sigma(r)|\left(|u(r)|^{q_{1}(r)-1}\left|u^{i}(r)\right|-\left|\mu^{i}\right|_{0}\left|(w(r))^{1 /(p(r)-1)} u^{\prime}(r)\right|^{q_{2}(r)}\right)+e^{i}(r)\right\} \\
& \quad \geq \Theta^{i}(r)\left[\sigma_{1} b_{1}+e^{i}(r)\right]>0 .
\end{aligned}
$$

It is a contradiction to (1.2). 
Assume that $\sigma u^{i}$ is negative, then

$$
\begin{aligned}
& f^{i}\left(r, u,(w(r))^{1 /(p(r)-1)} u^{\prime}\right) \\
& \quad \leq \Theta^{i}(r)\left[-|\sigma(r)|\left(|u(r)|^{q_{1}(r)-1}\left|u^{i}(r)\right|-\left|\mu^{i}\right|_{0}\left|(w(r))^{1 /(p(r)-1)} u^{\prime}(r)\right|^{q_{2}(r)}\right)+e^{i}(r)\right] \\
& \quad \leq \Theta^{i}(r)\left[-\sigma_{1} b_{1}+e^{i}(r)\right]<0 .
\end{aligned}
$$

It is a contradiction to (1.2).

(ii) Suppose that $\left|u^{i}\right|_{0} \leq 2 \theta$, then $\varepsilon / 2(E+1)<\theta / E \leq\left|(w(r))^{1 /(p(r)-1)}\left(u^{i}\right)^{\prime}\right|_{0} \leq \varepsilon$.

This implies that $\left|\left(w\left(r_{1}\right)\right)^{1 /\left(p\left(r_{1}\right)-1\right)}\left(u^{i}\right)^{\prime}\left(r_{1}\right)\right| \geq \varepsilon / 2(E+1)$ for some $r_{1} \in I$. Since $u \in \overline{\Omega_{\varepsilon}}$, it is easy to see that

$$
\left|\left(w\left(r_{1}\right)\right)^{1 /\left(p\left(r_{1}\right)-1\right)}\left(u^{i}\right)^{\prime}\left(r_{1}\right)\right| \geq \frac{\varepsilon}{2(E+1)}=\frac{N \varepsilon}{2 N(E+1)} \geq \frac{\left|\left(w\left(r_{1}\right)\right)^{1 /\left(p\left(r_{1}\right)-1\right)} u^{\prime}\left(r_{1}\right)\right|}{2 N(E+1)} .
$$

According to the boundary value condition, there exists a $r_{0}^{i} \in I$ such that

$$
w\left(r_{0}^{i}\right)\left|u^{\prime}\left(r_{0}^{i}\right)\right|^{p\left(r_{0}^{i}\right)-2}\left(u^{i}\right)^{\prime}\left(r_{0}^{i}\right)=0,
$$

then

$$
w(r)\left|u^{\prime}\right|^{p(r)-2}\left(u^{i}\right)^{\prime}(r)=\lambda \int_{r_{0}^{i}}^{r} f^{i}\left(t, u,(w(t))^{1 /(p(t)-1)} u^{\prime}\right) d t, \quad \forall r \in(0,+\infty) .
$$

Since $\sigma_{2}<b_{2}$, combining (4.11), we have

$$
\begin{aligned}
\frac{|\varepsilon / 2(E+1)|^{p\left(r_{1}\right)-1}}{2 N(E+1)} & \leq \frac{1}{2 N(E+1)} w\left(r_{1}\right)\left|\left(u^{i}\right)^{\prime}\left(r_{1}\right)\right|^{p\left(r_{1}\right)-1} \leq \frac{1}{2 N(E+1)} w\left(r_{1}\right)\left|u^{\prime}\left(r_{1}\right)\right|^{p\left(r_{1}\right)-1} \\
& \leq w\left(r_{1}\right)\left|u^{\prime}\left(r_{1}\right)\right|^{p\left(r_{1}\right)-2}\left|\left(u^{i}\right)^{\prime}\left(r_{1}\right)\right| \leq \lambda\left|\int_{r_{0}^{i}}^{r_{1}}\right| f^{i}\left(t, u,(w(t))^{1 /(p(t)-1)} u^{\prime}\right)|d t| \\
& \leq \int_{0}^{+\infty} \Theta^{i}(t)\left[\sigma_{2}\left(|N \varepsilon|^{q_{1}(t)}+|\mu||N \varepsilon|^{q_{2}(t)}\right)+\left|e^{i}\right|_{0}\right] d t \\
& \leq \sigma_{2} \int_{0}^{+\infty} \Theta^{i}(t)\left[\left(|N \varepsilon|^{q_{1}(t)}+\|\mu\|_{0}|N \varepsilon|^{q_{2}(t)}\right)+|\theta|^{q_{1}(t)}\right] d t \\
& <\frac{1}{2 N(E+1)} \inf _{t \in I}\left|\frac{\varepsilon}{2(E+1)}\right|^{p(t)-1} \leq \frac{1}{2 N(E+1)}\left|\frac{\varepsilon}{2(E+1)}\right|^{p\left(r_{1}\right)-1} .
\end{aligned}
$$

It is a contradiction. 
Summarizing this argument, for each $\lambda \in(0,1)$, the problem (4.7) with (1.2) has no solution on $\partial \Omega_{\varepsilon}$.

$\left(2^{0}\right)$ For any $u \in \partial \Omega_{\varepsilon} \cap \mathbb{R}^{N}$, without loss of generality, we may assume that $a^{i}=\varepsilon$ and $\sigma(t)>0$, then we have

$$
\begin{aligned}
\int_{0}^{+\infty} f^{i}(t, a, 0) d t & =\int_{0}^{+\infty} \Theta^{i}(t)\left(\sigma(t)|a|^{q_{1}(t)-1} \varepsilon+e^{i}(t)\right) d t \\
& \geq \int_{0}^{+\infty} \Theta^{i}(t)\left(\sigma_{1}|\varepsilon|^{q_{1}(t)-1} \varepsilon+e^{i}(t)\right) d t>0
\end{aligned}
$$

It means that $\omega(a)=0$ has no solution on $\partial \Omega_{\varepsilon} \cap \mathbb{R}^{N}$.

$\left(3^{0}\right)$ Let

$$
\begin{gathered}
h^{i}(t, a, \lambda)=\Theta^{i}(t)\left[\lambda\left(\sigma(t)|a|^{q_{1}(t)-1} a^{i}+e^{i}(t)\right)+(1-\lambda) a^{i} \text { sgn } \sigma(t)\right], \\
h(t, a, \lambda)=\left(h^{1}(t, a, \lambda), \ldots, h^{N}(t, a, \lambda)\right) .
\end{gathered}
$$

Denote

$$
\Phi(a, \lambda)=\int_{0}^{+\infty} h(t, a, \lambda) d t
$$

According to $\left(\mathrm{A}_{3}\right)$, it is easy to see that, for any $\lambda \in[0,1], \Phi(a, \lambda)=0$ does not have solution on $\partial \Omega_{\varepsilon} \cap \mathbb{R}^{N}$, then the Brouwer degree

$$
d_{B}\left[\omega, \Omega_{\varepsilon} \cap \mathbb{R}^{N}, 0\right]=d_{B}\left[\Phi(a, 1), \Omega_{\varepsilon} \cap \mathbb{R}^{N}, 0\right]=d_{B}\left[\Phi(a, 0), \Omega_{\varepsilon} \cap \mathbb{R}^{N}, 0\right] \neq 0
$$

This completes the proof.

Theorem 4.2. If $u$ is a solution of (1.1)-(1.2) which is given in Theorem 4.1, then

(i) $\left|u^{\prime}(t)\right| \leq C_{1} / w(t)^{1 /(p(t)-1)}, t \in(0,+\infty)$;

(ii) $|u(+\infty)-u(r)| \leq \int_{r}^{+\infty}\left(C_{2} / w(t)^{1 /(p(t)-1)}\right) d t$, as $r \rightarrow+\infty$;

(iii) $|u(r)-u(0)| \leq \int_{0}^{r}\left(C_{3} / w(t)^{1 /(p(t)-1)}\right) d t$, as $r \rightarrow 0^{+}$.

Proof. Since $\lim _{r \rightarrow+\infty} p(r)$ exists and $\lim _{r \rightarrow+\infty} p(r)>1, \lim _{t \rightarrow 0^{+}} w(t)\left|u^{\prime}\right|^{p(t)-2} u^{\prime}(t)$ and $\lim _{t \rightarrow+\infty} w(t)\left|u^{\prime}\right|^{p(t)-2} u^{\prime}(t)$ both exist and equal, we have $\left|w(t)^{1 /(p(t)-1)}\right| \leq C$, for all $t \in$ $(0,+\infty)$. Thus

(i) $\left|u^{\prime}(t)\right| \leq C_{1} / w(t)^{1 /(p(t)-1)}, t \in(0,+\infty)$;

(ii) $|u(+\infty)-u(r)|=\left|\int_{r}^{+\infty} u^{\prime}(t) d t\right| \leq \int_{r}^{+\infty}\left(C_{2} / w(t)^{1 /(p(t)-1)}\right) d t$, as $r \rightarrow+\infty$;

(iii) $|u(r)-u(0)|=\left|\int_{0}^{r} u^{\prime}(t) d t\right| \leq \int_{0}^{r}\left(C_{3} / w(t)^{1 /(p(t)-1)}\right) d t$, as $r \rightarrow 0^{+}$.

We completes the proof. 
Corollary 4.3. Assume that $\lim _{r \rightarrow+\infty} p(r)$ exists, $\lim _{r \rightarrow+\infty} p(r)>1$, and

$$
\begin{aligned}
& C_{4} \leq \frac{w(t)}{t^{\alpha}} \leq C_{5}, \quad \alpha>p(t)-1 \quad \text { as } t \longrightarrow+\infty, \\
& C_{6} \leq \frac{w(t)}{t^{\alpha}} \leq C_{7}, \quad \alpha<p(t)-1 \quad \text { as } t \longrightarrow 0^{+},
\end{aligned}
$$

then

(i) $\left|u^{\prime}(t)\right| \leq C_{8} / t^{\alpha /(p(t)-1)}, t \in(0,+\infty)$;

(ii) $|u(+\infty)-u(r)| \leq \int_{r}^{+\infty}\left(C_{9} / t^{\alpha /(p(t)-1)}\right) d t$, as $r \rightarrow+\infty$;

(iii) $|u(r)-u(0)| \leq \int_{0}^{r}\left(C_{10} / t^{\alpha /(p(t)-1)}\right) d t$, as $r \rightarrow 0^{+}$.

Similar to the proof of Theorem 4.1, we have the following.

Theorem 4.4. Assume that $f(t, x, y)=\Theta(t)\left[\sigma(t)|x|^{q_{1}(t)-2} x+\mu(t)|y|^{q_{2}(t)-2} y\right]$, where $q_{1}, q_{2}, \sigma, \mu \in$ $C(I, \mathbb{R})$ satisfy $\max _{t \in I} p(t)<q_{1}(t), q_{2}(t)$, for all $t \in I, \Theta(\cdot) \in L^{1}$. On the conditions of $\left(A_{1}\right)-\left(A_{4}\right)$, if $\delta=1$, then problem (1.1)-(1.2) possesses at least one solution.

On the typical case, we have the following.

Corollary 4.5. Assume that $f(t, x, y)=\Theta(t)\left[\sigma(t)|x|^{q_{1}(t)-2} x+\mu(t)|y|^{q_{2}(t)-2} y\right]$, where $q_{1}, q_{2}, \sigma, \mu \in$ $C(I, \mathbb{R})$ satisfy $\min _{t \in I} p(t) \leq q_{1}(t), q_{2}(t) \leq \max _{t \in I} p(t), \Theta(\cdot) \in L^{1}$. On the conditions of Theorem 4.1, then problem (1.1)-(1.2) possesses at least one solution.

\section{Acknowlegments}

This work is partly supported by the National Science Foundation of China (10701066 and 10671084) and China Postdoctoral Science Foundation (20070421107), the Natural Science Foundation of Henan Education Committee (2008-755-65), and the Natural Science Foundation of Jiangsu Education Committee (08KJD110007).

\section{References}

[1] E. Acerbi and G. Mingione, "Regularity results for a class of functionals with non-standard growth," Archive for Rational Mechanics and Analysis, vol. 156, no. 2, pp. 121-140, 2001.

[2] Y. Chen, S. Levine, and M. Rao, "Variable exponent, linear growth functionals in image restoration," SIAM Journal on Applied Mathematics, vol. 66, no. 4, pp. 1383-1406, 2006.

[3] L. Diening, "Maximal function on generalized Lebesgue spaces $L^{p(\cdot)}$," Mathematical Inequalities $\mathcal{E}$ Applications, vol. 7, no. 2, pp. 245-253, 2004.

[4] X.-L. Fan and Q.-H. Zhang, "Existence of solutions for $p(x)$-Laplacian Dirichlet problem," Nonlinear Analysis: Theory, Methods E Applications, vol. 52, no. 8, pp. 1843-1852, 2003.

[5] X.-L. Fan, H.-Q. Wu, and F.-Z. Wang, "Hartman-type results for $p(t)$-Laplacian systems," Nonlinear Analysis: Theory, Methods \& Applications, vol. 52, no. 2, pp. 585-594, 2003.

[6] X. Fan, Q. Zhang, and D. Zhao, "Eigenvalues of $p(x)$-Laplacian Dirichlet problem," Journal of Mathematical Analysis and Applications, vol. 302, no. 2, pp. 306-317, 2005.

[7] A. El Hamidi, "Existence results to elliptic systems with nonstandard growth conditions," Journal of Mathematical Analysis and Applications, vol. 300, no. 1, pp. 30-42, 2004.

[8] H. Hudzik, "On generalized Orlicz-Sobolev space," Functiones et Approximatio Commentarii Mathematici, vol. 4, pp. 37-51, 1976. 
[9] O. Kováčik and J. Rákosník, "On spaces $L^{p(x)}(\Omega)$ and $W^{k, p(x)}(\Omega)$, " Czechoslovak Mathematical Journal, vol. 41, no. 4, pp. 592-618, 1991.

[10] Q. Zhang, "Existence of positive solutions for elliptic systems with nonstandard $p(x)$-growth conditions via sub-supersolution method," Nonlinear Analysis: Theory, Methods \& Applications, vol. 67, no. 4, pp. 1055-1067, 2007.

[11] Q. Zhang, "Existence of positive solutions for a class of $p(x)$-Laplacian systems," Journal of Mathematical Analysis and Applications, vol. 333, no. 2, pp. 591-603, 2007.

[12] Q. Zhang, "Existence of solutions for weighted $p(r)$-Laplacian system boundary value problems," Journal of Mathematical Analysis and Applications, vol. 327, no. 1, pp. 127-141, 2007.

[13] Q. Zhang, Z. Qiu, and X. Liu, "Existence of solutions for a class of weighted $p(t)$-Laplacian system multipoint boundary value problems," Journal of Inequalities and Applications, vol. 2008, Article ID 791762, 18 pages, 2008.

[14] Q. Zhang, X. Liu, and Z. Qiu, "The method of subsuper solutions for weighted $p(r)$-Laplacian equation boundary value problems," Journal of Inequalities and Applications, vol. 2008, Article ID 621621, 19 pages, 2008.

[15] Q. Zhang, "Boundary blow-up solutions to $p(x)$-Laplacian equations with exponential nonlinearities," Journal of Inequalities and Applications, vol. 2008, Article ID 279306, 8 pages, 2008.

[16] M. Růžička, Electrorheological Fluids: Modeling and Mathematical Theory, vol. 1748 of Lecture Notes in Mathematics, Springer, Berlin, Germany, 2000.

[17] V. V. Zhikov, "Averaging of functionals of the calculus of variations and elasticity theory," Izvestiya Akademii Nauk SSSR. Seriya Matematicheskaya, vol. 29, no. 1, pp. 33-66, 1987.

[18] Y. Guo, W. Shan, and W. Ge, "Positive solutions for second-order $m$-point boundary value problems," Journal of Computational and Applied Mathematics, vol. 151, no. 2, pp. 415-424, 2003.

[19] Y. Wang and W. Ge, "Existence of multiple positive solutions for multipoint boundary value problems with a one-dimensional p-Laplacian," Nonlinear Analysis: Theory, Methods E Applications, vol. 67, no. 2, pp. 476-485, 2007.

[20] Y. Wang and W. Ge, "Multiple positive solutions for multipoint boundary value problems with onedimensional p-Laplacian," Journal of Mathematical Analysis and Applications, vol. 327, no. 2, pp. 13811395, 2007.

[21] Y. Wang and W. Ge, "Positive solutions for multipoint boundary value problems with a onedimensional p-Laplacian," Nonlinear Analysis: Theory, Methods E Applications, vol. 66, no. 6, pp. 1246 1256, 2007. 